\title{
Antinociceptive and anti-inflammatory effects of thiamine in Long Evans rats
}

\author{
Sayema Ainan ${ }^{1}$, Noorzahan Begum ${ }^{2}$, Taskina Ali $^{3}$
}

\begin{abstract}
Background: Thiamine along with other B vitamins has been prescribed since long for treatment of various painful conditions, though individual effects of thiamine on nociception and inflammation are yet to be clearly demonstrated. Objective: To assess the effects of increasing doses of thiamine supplementation against pain and inflammation. Methods: This experimental study was conducted in the Department of Physiology, Bangabandhu Sheikh Mujib Medical University (BSMMU), Dhaka from March 2014 to February 2015. Total twenty four male Long Evans rats weighing ( $200 \pm 20 \mathrm{gm}$ ) were treated with three different doses of thiamine hydrochloride (THCL 100, 200, 250, mg/kg/day; experimental) or normal saline ( $5 \mathrm{ml} / \mathrm{kg} /$ day; control) intraperitoneally for 7 consecutive days. To evaluate the thiamine's effect on nociceptive pain, early phase (0-5 minutes) and on inflammatory pain, late phase (16-60 minutes) of the formalin test, were observed. In both phases, total frequency of jerking and total duration of flexing and licking of the right hind paw were counted after administration of subcutaneous formalin $(50 \mu 1,2.5 \%)$ injection. After formalin test, all the rats were also subjected to formalin induced paw edema test using a water plethysmometer to observe the antiinflammatory effect of thiamine. Statistical analysis was done by ANOVA, followed by Bonferroni post hoc test. In the interpretation of results, $\mathrm{p} \leq 0.05$ was considered as significant. Results: In formalin test, thiamine lowered frequency of jerking (after all 3 doses, in both phases) and duration of flexing and licking $(200 \mathrm{mg} / \mathrm{kg}-\mathrm{p}<0.001$, in late phases; $250 \mathrm{mg} / \mathrm{kg}$ ( $\mathrm{p}<0.001)$, in both phases) significantly. Additionally, thiamine lowered paw edema significantly $(\mathrm{p}<0.001)$ in higher 2 doses. Conclusion: This study concludes that, thiamine may have dose dependent antinociceptive and anti-inflammatory effects.
\end{abstract}

Key words: Thiamine, antinociceptive, anti-inflammation, formalin test, paw edema test.

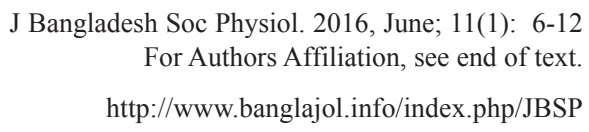

http://www.banglajol.info/index.php/JBSP

\section{Introduction}

A mong the water soluble vitamin B complex, Thiamine $\left(\mathrm{B}_{1}\right)$ was isolated in 1930s and was one of the first organic compounds to be recognized as a vitamin ${ }^{1}$. It was first named "Aneurin" (antineuritic vitamin) $)^{2}$ and is one of the most important medication needed in basic health

Received 6th Jan. 2016; Accepted 3rd May 2016

J Bangladesh Soc Physiol. 2016, June; 11(1): 6-12 system $^{3}$ in the present world. It is used in biosynthesis of different neurotransmitters, such as, acetylcholine and gamma-aminobutyric acid (GABA) in addition to its role as a coenzyme ${ }^{4}$. Thiamine deficiency may cause insomnia, fatigue, constipation, gastrointestinal distress, muscular atrophy, cramps in addition to beriberi (Wernicke-Korsakoff syndrome) with central as 
well as peripheral nervous and cardiovascular symptoms leading to death ${ }^{5}$.

Thiamine with $\mathrm{B}_{6}$ and $\mathrm{B}_{12}$, have been prescribed for long, either alone or with other analgesics, for treatment of various painful conditions, which are not associated with their deficiencies, such as polyneuropathy, neuritis, painful vertebral syndrome, rheumatic diseases, carpal tunnel syndrome as well as premenstrual tension ${ }^{6-8}$. However, it has been proposed that, high therapeutic doses of $\mathrm{B}$ vitamins might be needed to be administered to exert their complete analgesic, antinociceptive or anti-inflammatory effects $^{9-11}$. Several studies have shown that high doses of thiamine with vitamin $\mathrm{B}_{6}$ and $\mathrm{B}_{12}$, potentiate the antinociceptive effect of diclofenac, ketorolac, ketoprofen as well as acetaminophen $^{12-15}$. Furthermore, in several animal studies combined thiamine, B6 and B12 have been found effective to reduce nociceptive and inflammatory pain, both after single (acute) as well as after chronic administrations (for ${ }^{3}$ 7 consecutive days) $)^{9,13,15-19}$.

It is noteworthy that, rats treated with high doses of $B$ vitamins have not shown any toxic effects ${ }^{20}$. Moreover, repetitive (chronic) administrations of the $B$ vitamins produced long term inhibition of both severity and duration of pain ${ }^{20}$. Recently, the analgesic and anti-inflammatory effects of thiamine have been demonstrated in different types of animals and the acute and chronic antinociceptive effect of thiamine was evaluated by hot plate test in mice model ${ }^{21,22}$. In addition, $125 \mathrm{mg} / \mathrm{kg} /$ body weight of thiamine decreased acute inflammation in the animals' ear induced by xylene ${ }^{21}$. However, Franca et al. have shown that, thiamine at chronic (for ${ }^{3} 7$ consecutive days administration) doses with 50 and $100 \mathrm{mg} / \mathrm{kg}$ / body weight, reduced pain in the second phase (16th to 30th minutes) of formalin test ${ }^{16}$. Similar trend of decrement in the inflammatory pain after administration of different doses of combination of thiamine, B6 and B12 have been reported in several studies ${ }^{13,15,17,18}$.
Formalin test is one of the valid and reliable animal models to assess both the nociceptive as well as inflammatory form of pain and is sensitive to both centrally as well as peripherally acting analgesics $^{23,24}$. Here, subcutaneous injection of formalin at hindpaw induces inflammation, which leads to a response characterized by jerking, flexing followed by licking of the affected hindlimb $23,25,26$. Pain intensity is measured by converting these behavioral responses into numerical values $24,25,26,27$, assessed in early phase (first 5-7 minutes, results from direct chemical stimulation of the nociceptive afferent fibers mainly $\mathrm{C}$ fibers $)^{28}$, while the late phase (last 40-45 minutes, results from the action of locally released inflammatory mediators and also by the facilitation of synaptic transmission in thespinalcord) ${ }^{29}$.

In addition, the formalin induced paw edema test in rodents is a very simple, accurate and one of the common models for the assessment of inflammation in animals $30,31,32$, where the characteristic hindpaw edema has been associated with increased amount of chemical mediators in tissue fluids ${ }^{31}$.

In recent years, various researchers have intended to find out alternatives to the conventional analgesics in order to replace them with natural substances, or to decrease their doses to minimize their adverse effects. Though antinociceptive and anti-inflammatory effects of thiamine with different doses and duration were reported but there is lack of information about the potency of aforementioned effects with higher doses along with chronic administration of this vitamin. Therefore, this study was designed to evaluate the antinociceptive as well as anti-inflammatory effects of chronic (single daily dose for 7 consecutive days) supplementations of thiamine in increasing doses $(100,200$ and $250 \mathrm{mg} / \mathrm{kg}$ of body weight) by formalin test and formalin induced paw edema test, in rats. 


\section{Methods}

This experimental study was conducted in the Department of Physiology, Bangabandhu Sheikh Mujib Medical University (BSMMU), Dhaka from March 2014 to February 2015, with prior protocol approval from the Institutional Review Board of the University.

Procurement and maintenance of animals $\mathrm{s}^{33,34}$ : Twenty four (24) male Long Evans rats weighing 180 to $220 \mathrm{gm}$ were obtained from animal house of Bangladesh Institute of Research and Rehabilitation for Diabetes, Endocrine and Metabolic Disorders (BIRDEM), Shahbag, Dhaka, and were kept under a 12/12 hour light/ dark cycle in a room with constant temperature $(28 \pm 50 \mathrm{C})$. The animals were acclimatized for 7 consecutive days prior to the experiments and had free access to standard laboratory food and cooled boiled water. The experiments were performed during the day time between 8:00 to 14:00 hours, to avoid any circadian influences. All experiments and animal care were performed according to the guidelines set in the 'Manual for Care and Use of Laboratory Animals' by the Animal Experimentation Ethics Committee (AEEC) of the International Centre for Diarrhoeal Disease Research, Bangladesh (icddr,b).

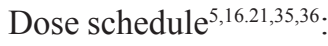

Experimental solutions of thiamine (100, 200, $250 \mathrm{mg} / \mathrm{kg}$ ) were prepared by dissolving desired amounts of thiamine hydrochloride (THCL) in 5 $\mathrm{ml} / \mathrm{kg}$ of normal saline (NS). All compounds (THCL for experimental animals and NS for control animals) were administered intraperitoneally for 7 consecutive days and the last dose was administered 1 hour prior to the experiments.

Formalin test $\mathrm{t}^{16,20,22,30,37-38}$ :

The rats were acclimatized in the experimental laboratory environment, in the observation cage of the plexiglass formalin box $(30 \times 30 \times 30 \mathrm{~cm} 3)$ for 1 hour daily (within 8:00 to 14:00 hours) for 7 consecutive days prior to the formalin test. Then on the day of experiment (Day 7), 1 hour after last dose of (i.p.) injection of THCL or equal volume of NS, the rats were restrained by a thick towel and $50 \mu \mathrm{l}$ of dilute formalin (2.5\%) was injected subcutaneously into the plantar aspect of the rats' right hindpaw with an insulin syringe. Immediately thereafter, the animal was placed in the observation cage of the formalin box and the pain behaviors were observed for 60 consecutive minutes. Within this time the first 5 minutes (1st -5 th) was considered as the early phase, and last 45 minutes (16th -60 th) as the late phase. Observation was made by counting the total frequency of jerking and total duration of flexing plus licking of the injected paw during this time through a mirror fixed below the formalin box at 45 Ú angle. A stop watch was used to note the time.

Formalin induced paw edema test ${ }^{16,31,39}$ :

Anti-inflammatory effect of thiamine was determined by the formalin induced paw edema model, where the amount of paw edema resulted from intra-planter injection of $50 \mu 1$ of $2.5 \%$ formalin was used an indicator of inflammation severity. Following 60 minutes of recording the pain behaviors (about 120 minutes after the i.p administration of THCL or NS), the animal was sacrificed. Then the volumes of the animal's right and left hind paws were measured by using a water plethysmometer. Left paw volume was subtracted from right paw volume to attain the net edema volume.

Data were expressed as mean \pm SEM and statistically analyzed by SPSS (version 17.0) using analysis of variance (ANOVA), followed by a Bonferroni Post hoc test. In the interpretation of results, $\mathrm{p}$ value $\leq 0.05$ was considered as significant.

\section{Results}

In this study, THCL significantly reduced the jerking frequency ( $\mathrm{p} \leq 0.001$, in all doses) and flexing and licking duration $(\mathrm{p} \leq 0.001$, in $250 \mathrm{mg} / \mathrm{kg}$ ) in the early phase of formalin test (Figure 1).

In addition, this vitamin also reduced the jerking frequency ( $\mathrm{p} \leq 0.001$, in all doses) as well as the duration of flexing and licking $(\mathrm{p} \leq 0.001$, in 200 

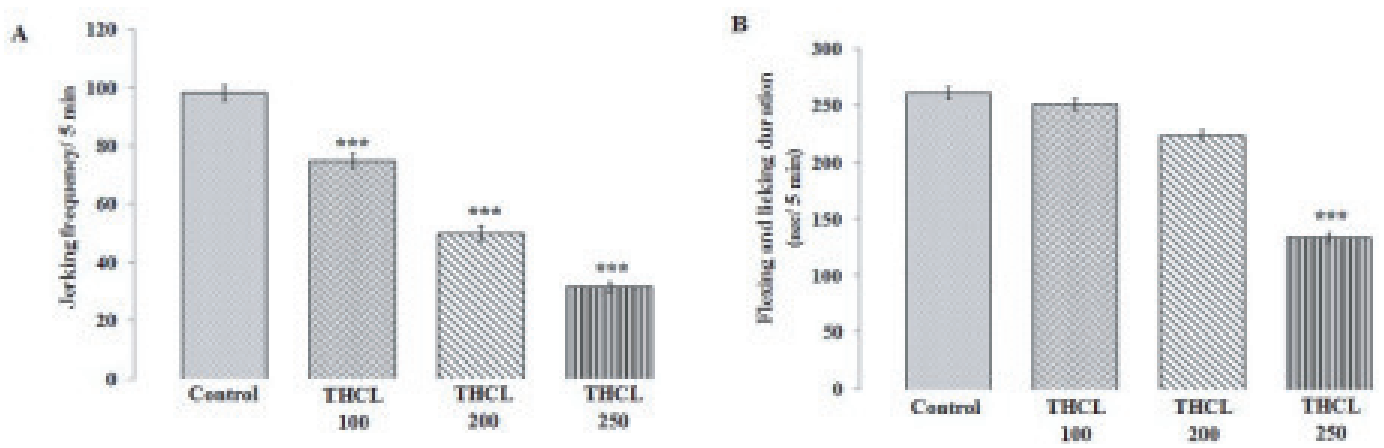

Figure 1: Antinociceptive effects of thiamine hydrochloride (THCL) in the early phase of formalin test (1st- $5^{\text {th }}$ minutes). THCL (at100, 200 and $250 \mathrm{mg} / \mathrm{kg}$ doses) lowered both jerking (A) and flexing and licking (B) responses. Each bar symbolizes for mean \pm SE for 6 rats. ${ }^{* * *}=\mathrm{p}<0.001$, compared to control.
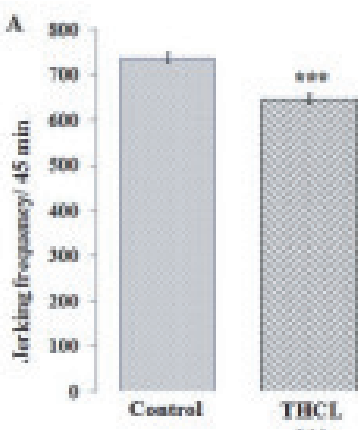

100

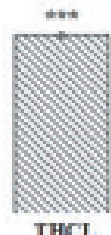

THCL
200
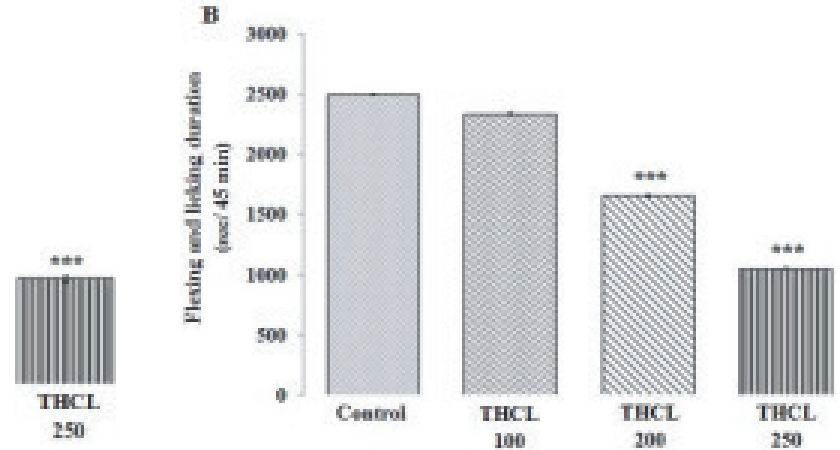

Figure 2: Antinociceptive effects of thiamine hydrochloride (THCL) in the late phase of formalin test (16th-60th minutes). THCL (at 100, 200 and $250 \mathrm{mg} / \mathrm{kg}$ doses) lowered both jerking (A) as well as flexing and licking (B) responses. Each bar symbolizes for mean \pm SE for 6 rats. $* * *=p<0.001$, compared to control.

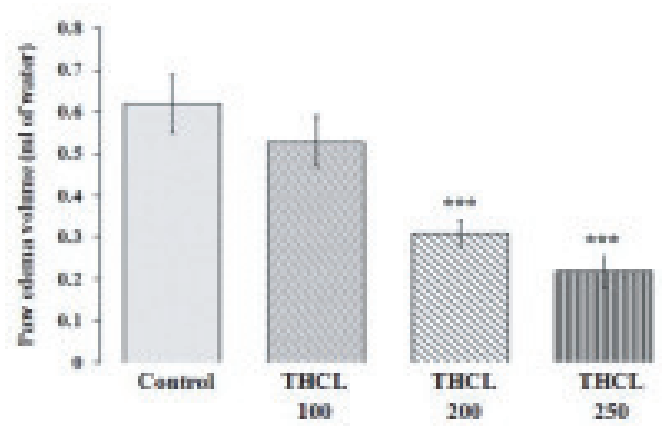

Figure 3: Anti-inflammatory effects of thiamine hydrochloride (THCL at 100, 200 and $250 \mathrm{mg} /$ $\mathrm{kg}$ doses) in formalin induced paw edema model. Each bar represents for mean \pm SE for 6 rats. $* * *=p<0.001$, compared to control. and $250 \mathrm{mg} / \mathrm{kg}$ ) in the late phase of formalin test (Figure 2).

Moreover in the outcome of the paw edema test, THCL showed statistically significant $(\mathrm{p}<0.001)$ decrement in edema volume after 200 and 250 $\mathrm{mg} / \mathrm{kg}$ in the paw edema model (figure 3 ).

\section{Discussion}

In this study, thiamine supplementation in chronic (consecutive 7 days) administration elicited potent antinociceptive effects, as evidenced by significant lowering of jerking frequency as well as duration of flexing and licking in both phases of formalin test in experimental rats. Similar trend 
of findings has been observed by Franca et al. ${ }^{16}$, though they investigated the effect of administration of individual thiamine as well as combined (20:20:1) thiamine, pyridoxine and cyanocobalamine (TPC), both at 50 and $100 \mathrm{mg} /$ $\mathrm{kg}$ (i.p.). Furthermore decrement of pain response was also reported by other researchers after single oral administration of TPC at different doses in different pain models ${ }^{13,18,19}$.

Again, in this study, chronic supplementation of $\mathrm{B}_{1}(200$ and $250 \mathrm{mg} / \mathrm{kg})$ significantly lowered the edema in the formalin induced paw edema test. Similar observations were reported by Moallem and his colleagues after single supplementation of $\mathrm{B}_{1}$ alone ${ }^{21}$ and also by Franca et al. after chronic supplementation of combined $\mathrm{B}_{1}, \mathrm{~B}_{12}$ and $\mathrm{B}_{6}$ in mice models in different dosage from this study ${ }^{16}$.

The exact possible mechanisms regarding these observed effects cannot not be revealed directly from the present study. However, several investigators of different countries proposed various suggestions on these aspects, which might be cause of our present findings. It has been suggested that, antinociceptive effect of THCL may be due to increase inhibitory control of afferent nociceptive neurons at the spinal cord along with decrease response of thalamic neurons to nociceptive stimulation ${ }^{40,41}$. Moreover, thiamine supplementation may also increase the availability or effectiveness of serotonin as well as noradrenaline in the $\mathrm{CNS}^{24,26,27}$. Besides the central effects in lowering nociception, it has been proposed that peripheral anti-inflammatory effect of this vitamin may be mediated through regulation of arachidonic acid pathway in macrophage, where it can block the expression of any of the enzymes (cycloxygenase-2, lipoxygenase-2, thromboxane B synthase, prostacycline synthase) as well as transcription factor nuclear factor kappa ${ }^{28}$. In addition, this vitamin may also decrease the oxidative stress and production of free radicals ${ }^{29}$.

As, all 3 doses of THCL(100, 200 and $250 \mathrm{mg} /$ $\mathrm{kg}$ ) in our study significantly lowered the early and late phases of formalin test, it is suggestive that all those doses possess both the central and peripheral antinociceptive effects. Along with this, significant reduction of formalin induced hind paw edema after THCL administration, suggested its probable role in amelioration of inflammation and thereby inflammatory pain with higher two doses of (200 and $250 \mathrm{mg} / \mathrm{kg}$ ). In addition all these effects of $B_{1}$ on lowering pain and inflammation in the present study were related with the dose of supplementation, as evidenced by more decrement of all the study variables in the higher dose $(250 \mathrm{mg} / \mathrm{kg} / \mathrm{day})$ group. It is suggested that, chronic supplementation at progressively higher doses might cause more accumulation of $B_{1}$ in the serum which probably exerted a cumulative effect for greater decrement of pain and inflammatory variables in our study. Strikingly, the lowest dose $(100 \mathrm{mg} / \mathrm{kg}$ ) of THCL used in our study did not lower the paw edema significantly, whereas it inhibited the jerking frequency significantly in the late phase of formalin test. These findings incite our thinking that $\mathrm{B}_{1}$ may possess any other mechanism of decrement of inflammatory pain other than anti-inflammation.

\section{Conclusion}

In conclusion, it is recommended that, chronic thiamine supplementation may diminish both the acute and chronic forms of nociception and may have potent role against inflammation, which are dose dependent. However, further experimental study is recommended to elucidate the exact mechanism responsible for these effects.

\section{Conflict of interest: none}

\section{Authors' affiliation}

1. *Sayema Ainan, Assistant Professor, Department of Physiology, Gazi Medical College, Khulna. Email: sayema.ainan@gmail.com.

2. Noorzahan Begum, Professor, Department of Physiology, Bangabandhu Sheikh Mujib Medical University, Dhaka. Email: noorzahan52@gmail.com.

3. Taskina Ali, Associate Professor, Department of Physiology, Bangabandhu Sheikh Mujib Medical University, Dhaka. Email: taskinadr@gmail.com.

*for correspondence 


\section{References}

1. Rindi G. Thiamine. In: Ziegler EE, Filer Jr. LJ, editors. Present Knowledge in Nutrition. Washington D.C: International life science institute press; 1996. p .11601166.

2. Carpenter KJ. Beriberi, white rice, and vitamin B: a disease, a cause, and a cure. Berkeley: University of California Press; 2000.

3. World Health Organization. WHO list of essential medicines. http://apps.who.int/iris/bitstream (2013). Accessed 29 May 2014.

4. Mahan LK, Escott-Stump S, editors. Krause's food, nutrition \& diet therapy.10th ed. Philadelphia: W.B. Saunders Company; 2000.

5. Beers MH, Porter RS, Jones TV, Kaplan JL, Berkwits $\mathrm{M}$, editors. The Merck manual of diagnosis and therapy.18th ed. New Jersey: Merck Research Laboratories; 2006.

6. Brüggemann $\mathrm{G}$, Koehler CO, Koch EM. Results of a double-blind study of diclofenac + vitamin B1, B6, B12 versus diclofenac in patients with acute pain of the lumbar vertebrae. A multicenter study. Wien Klin Wochenschr. 1990;68(2):116-120.

7. Bernstein AL, Dinesen JS. Brief communication: effect of pharmacologic doses of vitamin B6 on carpal tunnel syndrome, electroencephalographic results and pain. J Am Coll Nutr. 1993;12(1): 73-76.

8. Wyatt KM, Dimmock PW, Jones PW, Shaughn O'Brien PM. Efficacy of vitamin B6 in the treatment of premenstrual syndrome: systematic review. Br Med J. 1999;318:1375-1381.

9. Bartoszyk GD, Wild A. Antinociceptive effects of pyridoxine, thiamine, and cyanocobalamin in rats. Ann N Y Acad Sci.1990;585:473-476.

10. Kuwabara S, Nakazawa R, Azuma N, Suzuki M, Miyajima K, Fukutake T, Hattori T. Intravenous methylcobalamin treatment for uremic and diabetic neuropathy in chronic hemodialysis patients. Intern Med. 1999;38(6):472-475.

11. Mauro GL, Martorana U, Cataldo P, Brancato G, Letizia G. Vitamin B12 in low back pain: a randomised, double-blind, placebo-controlled study. Eur Rev Med Pharmacol Sci. 2000;4:53-58.

12. Reyes-García G, Medina-Santillán R, Terán-Rosales F, Mateos-García E, Castillo-Henkel C. Characterization of the potentiation of the antinociceptive effect of diclofenac by vitamin B complex in the rat. J Pharmacol Toxicol Methods. 1999;42(2):73-7.

13. Medina-Santillan R, Reyes-Garcia G, Rocha-Gonzalez HI, Granados-Soto V. B Vitamins Increase the Analgesic Effect of Ketorolac in the Formalin Test in the Rat. Proc West Pharmacol Soc. 2004;47:95-99.

14. Rodriguez-Silverio J, Aguilar-Carrasco JC, Reyes-Garcia G, Medina-Santillan R, Flores-Murrieta FJ. Antinociceptive and antiinflammatory effects of ketoprofen are potentiated by a vitamin B mixture in the rat. Drug Dev Res. 2005;64:66-70.

15. Teran-Rosales F, Medina-Santillan R, Reyes-Garcia G, Grandos-Soto V. Synergistic antinociceptive interaction between acetaminophen or metamizol and B vitamins in the formalin test. Drug Develop Res. 2006;66:286-94.

16. Franca DS, Souza ALS, Almeida KR, Dolabella SS, Martinelli C, Coelho MM. B vitamins induce an antinociceptive effect in the acetic acid and formaldehyde models of nociception in mice. Eur $\mathrm{J}$ Pharmacol. 2001;421(3):157-64.

17. Reyes-García G, Medina-Santillán R, Terán-Rosales F, Castillo-Henkel C, Rodríguez-Silverio J, TorresLópez JE, Ochoa-Cetina L, Medina-Tato DA, Granados-Soto V. Analgesic effect of B vitamins in formalin-induced inflammatory pain. Proc West Pharmacol Soc. 2001;44:139-140.

18. Reyes-García G, Castillo-Henkel C, Medina-Santillán R, Terán-Rosales F, Mateos-García E,Grandos-Soto V. Mechanism of Analgesic Action of B Vitamins in Formalin Induced Inflammatory Pain. Proc West Pharmacol Soc. 2002;45:144-146.

19. Rocha-Gonzalez HI, Teran-Rosales F, Reyes-Garcia G, Medina-Santillan R, Granados-Soto V. B vitamins increase the analgesic effect of diclofenac in the rat. Proc West Pharmacol Soc. 2004;47:84-87.

20. Wang Z-B, Gan Q, Rupert RL, Zeng Y-M, Song X-J. Thiamine, pyridoxine, cyanocobalamin and their combination inhibit thermal, but not mechanical hyperalgesia in rats with primary sensory neuron injury. Pain. 2005;114:266-277.

21. Moallem SA, Hosseinzadeh H, Farahi, S. A Sudy of Acute and Chronic Anti-nociceptive and Antiinflammatory Effects of Thiamine in Mice. Iran Biomed J. 2008;12(3):173-178.

22. Abbott FV, Ocvirk R, Najafee R, Franklin KBJ. Improving the efficiency of the formalin test. Pain. 1999;83:561-569. 
23. Barrot M. Tests and models of nociception and pain in rodents. Neurosci. 2012;211:39-50.

24. Dakshinamurti K, Sharma SK, Bonke D. Inûuence of $\mathrm{B}$-vitamins on binding properties of serotonin receptors in the CNS of rats. Wien Klin Wochenschr. 1990;68:42-145.

25. Heapy CG, Jamieson A, Russel NJW. Afferent C-fiber and A-d activity in models of inflammation. Br J Pharmacol. 1987; 90:164.

26. Jurna I. Analgesic and analgesia-potentiating action of B vitamins. Schmerz.1998;12(2):136-41.

27. Dimpfel W, Spuler M, Bonke D. B vitamin induced effects on noradrenergic and serotonergic controlled neuronal activity in the rat brain. Ann N Y Acad Sci. 1990;585:480-82.

28. Shoeb M, Ramana KV. Anti-Inflammatory Effects of Benfotiamine are Mediated Through the Regulation of Arachidonic Acid Pathway in Macrophages. Free Radic Biol Med. 2012;52(1):182-190.

29. Mehta R, Dedina L, O’Brien PJ. Rescuing hepatocytes from iron-catalyzed oxidative stress using vitamins B1 and B6. Toxicol In Vitro. 2011;25:1114-1122.

30. Yashpal K, Coderre TJ. Influence. of formalin concentration on the antinociceptive effects of antiinflammatory drugs in the formalin test in rats: separate mechanisms underlying the nociceptive effects of lowand high-concentration formalin. Eur J Pain. 1998;2(1):63-68.

31. Fereidoni M, Ahmadiani A, Semnanian S, Javan M. An accurate and simple method for measurement of paw edema. J Pharmacol Toxicol Method. 2000;43: 11-14.

32. Vogel HG, editor. Drug discovery and evaluation: pharmacological assays. New York: Springer; 2002.
33. Uddin Z, Aninda KN, Anowara J, Mycal D, Masud MM, Talha BE. Analgesic activities of Crinum asiaticum. Mol Clin Pharmacol. 2012;3(2):125-33.

34. Islam KMN, Rahman ASMH, Al-Mahmud KA. Manual for care and use of laboratory animals. Animal resources branch. International Centre for Diarrhoeal Diseases Research, Bangladesh; 2001

35. Bitsch R. Vitamin B1 (Thiamine). In: Biesalski HK, Schrezenmeir J, Weber P, Weiss HE, editors. Vitamine - Physiologie, Pathophysiologie, Therapie. Stuttgart: Georg Thieme Verlag; 1997. p.67-74.

36. Royer-Morrot MJ, Zhiri A, Paille F, Royer RJ. Plasma thiamine concentrations after intramuscular and oral multiple dosage regimens in healthy men. Eur J Clin Pharmacol. 1992;42:219-222.

37. Hagiwara H, Kimura F, Mitsushima D, Funabashi T. Formalin-induced nociceptive behavior and c-Fos expression in middle-aged female rats. Physiol Behav. 2010;100:101-104.

38. Abbott FV, Franklin KBJ, Westbrook, RF. The formalin test: scoring properties of the first and second phases of the pain response in rats. Pain. 1995;60:91-102.

39. Ali T, Javan M, Sonboli A, Semnanian S. Evaluation of the antinociceptive and anti-inflammatory effects of essential oil of Nepeta pogonosperma Jamzad et Assadi in rats. DARU J Pharm Sci. 2012; 20: 1-8.

40. Sharma SK, Bolster B, Dakshinamurti K. Effects of pyridoxine on nociceptive thalamic unit activity. Ann N Y Acad Sci. 1990;585:549-553.

41. Jurna I, Carlsson KH, Bonke D, Fu QG, Zimmermann M. Suppression of thalamic and spinal nociceptive neuronal response by pyridoxine, thiamin and cyanocobalamin. Ann N Y Acad Sci.1990;585:492495. 